$$
\mu_{0}=\sqrt{\frac{\left[\lambda_{0} \lambda_{0}\right]+[a \delta][\alpha \delta]+[b \delta][\beta \delta]+[c \delta][\gamma \delta]+\cdots-\frac{\rho}{\pi}[\delta \delta]}{\pi-\rho}}
$$

Man kann diesen Ausdruck noch auf eine einfachere Form bringen. Wir schreiben zu diesem Zweck die Gleichungen (2), indem wir beachten, dass $l-v+\delta=l_{0}-v$ ist, folgendermassen:

$$
\left\{\begin{array}{l}
\delta=a x+b y+c z+\cdots+b_{0}-v \\
\delta^{\prime}=a^{\prime} x+b^{\prime} y+c^{\prime} z+\cdots+b_{0}^{\prime}-v^{\prime} \\
\delta^{\prime \prime}=a^{\prime \prime} x+b^{\prime \prime} y+c^{\prime \prime} z+\cdots+l_{0}^{\prime \prime}-\tau^{\prime \prime} \\
\text { etc. }
\end{array}\right.
$$

Wenn wir nun die $\delta$ für den Augenblick als die wirklichen Beobachtungsfehler ansehen, so können wir nach der Methode der kleinsten Quadrate aus (14) fur die Unbekannten $x, y, z, \ldots$ gewisse Werthe $\mathfrak{A}, \mathcal{H}, \mathfrak{C}, \ldots$ finden, nämlich (Art. 24)

$$
\begin{aligned}
& \mathfrak{A}=-\Sigma \alpha\left(l_{0}-v\right) \\
& \mathfrak{Y}=-\Sigma \beta\left(l_{0}-v\right) \\
& \mathfrak{C}=-\Sigma \gamma\left(l_{0}-v\right) \\
& \text { etc. }
\end{aligned}
$$

Nach analogen Formeln ist aber

$$
\begin{aligned}
& A_{0}=-\sum \alpha l_{0} \\
& B_{0}=-\sum \beta l_{0} \\
& C_{0}=-\sum y l_{0} \\
& \text { etc. }
\end{aligned}
$$

$$
\text { und nach (3) } \begin{aligned}
x-A & =\Sigma a v \\
y-B & =\Sigma \beta v \\
z-C & =\Sigma y v \\
\text { etc. } &
\end{aligned}
$$

wodurch

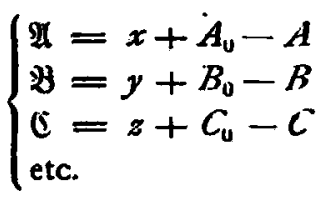

Berlin 8888 Dec. 22.
Setzen wir diese Werthe statt $x, y, z, \ldots$ in die Bedingungsgleichungen ein, so erhalten wir die ubrigbleibenden Fehler $x, x^{\prime}, x^{\prime \prime}, \ldots$, nämlich

$$
\begin{aligned}
& x=a \mathfrak{U}+b \mathfrak{B}+c \mathfrak{E}+\cdots+l_{0}-v \\
& x^{\prime}=a^{\prime} \mathfrak{A}+b^{\prime} \mathfrak{B}+c \mathfrak{E}+\cdots+b_{0}^{\prime}-v^{\prime} \\
& x^{\prime \prime}=a^{\prime \prime} \mathfrak{A}+b^{\prime \prime} \mathfrak{H}+c^{\prime \prime} \mathbb{C}+\cdots+l_{0}^{\prime \prime}-v^{\prime \prime} \\
& \text { etc. }
\end{aligned}
$$

oder, wenn man die Werthe (15) einfuhrt und (2), (8) und (9) berücksichtigt,

$$
\left\{\begin{array}{l}
x=\lambda_{0}-\hat{\lambda} \\
x^{\prime}=\lambda_{0}^{\prime}-\hat{\lambda}^{\prime} \\
x^{\prime \prime}=\lambda_{0}{ }^{\prime \prime}-\lambda^{\prime \prime} \\
\text { etc. }
\end{array}\right.
$$

Zwischen den wirklichen Fehlern $\delta$ und den nach der Methode der kleinsten Quadrate gefundenen ubrig bleibenden Fehlern $x$ besteht aber immer eine der Gleichung (I analoge Relation, namlich

$$
[x \boldsymbol{x}]=[\delta \delta]-[a \delta][a \delta]-[b \delta][\beta \delta]-[c \delta][\gamma \delta]-\cdots
$$

Hiernach erhalten wir unter Berücksichtigung von (I6)

$$
\mu_{0}=\sqrt{\frac{\left[\lambda_{0} \lambda_{0}\right]}{\pi-\rho}+\frac{[\delta \delta]}{\pi}-\frac{\left[\left(\lambda_{0}-\lambda\right)\right.}{\pi-\rho} \overline{\left.\left(\lambda_{0}-\lambda\right)\right]}} .
$$

Die Glieder $\frac{[\delta \delta]}{\pi}-\frac{\left[\left(\lambda_{0}-\lambda\right)\left(\lambda_{0}-\lambda\right)\right]}{\pi-\rho}$. wird man im Allge: meinen wegen der Unbekanntschaft mit dem Gesetze der Fehlervertheilung und den wahren Werthen der Unbekannten nicht streng bestimmen können. Man wird sich unter Um. ständen aber in zahnlicher Weise helfen können, wie es Hr. Kleiber in seinem oben angefuhrten Aufsatze gethan hat.

Fuir die Praxis wird wohl stets an der Berulcksichtigung jener Glieder wenig genug gelegen sein; für uns kam es nur darauf an, ihre theoretische Existenz nachzuweisen.

\title{
R. Lèhmann-Filhés.
}

\section{Auszug aus einem Schreiben von Hrn. Geo. C. Comstock, Director des Washburn Observatory betr. die Sonnenfinsterniss 1889 Jan. I.}

I olserved the first contact of the eclipse yesterday with the $15 \%$ inch. equatorial of this observatory, aperture reduced to six inches, power 195 . The sky was clear but seeing rather poor. Contact was observed at $3^{\mathrm{h}} 19^{\mathrm{m}} 54^{\mathrm{s}}$ Madison M. T. This time is probably $1^{3}$ late and may be as much as $2^{3}$ too late.

Madison 1889 Jan. 2.

Geo. C. Comstock. 